\section{European Specialist in Clinical Chemistry and Laboratory Medicine}

H. Baum

Institut für Laboratoriumsmedizin, Mikrobiologie und Blutdepot, Regionale Kliniken Holding RKH GmbH, Ludwigsburg, Deutschland

\section{Synonym(e) Eur Clin Chem}

Beschreibung Die Anerkennung als „European Specialist in Clinical Chemistry and Laboratory Medicine" wird durch die Registrierungskommission der $>$ European Communities Confederation of Clinical Chemistry (EC4-RC) vergeben. Dabei wird von EC4-RC überprüft, ob die Weiterbildungsin- halte im jeweiligen Mitgliedsstaat den von der Registrierungskommission erarbeiteten Mindeststandards entspricht. Die Inhalte sind in einem Syllabus zusammengefasst, wobei auch die speziellen Bedingungen der einzelnen Mitgliedsstaaten berücksichtigt werden. Voraussetzung für die Anerkennung ist die Registrierung im jeweiligen Mitgliedsstaat. Für Deutschland ist dies die Registrierung als „Klinische Chemikerin/Klinischer Chemiker" bei der $\triangleright$ Deutsche Gesellschaft für Klinische Chemie und Laboratoriumsmedizin e.V. (DGKL).

\section{Literatur}

www.eflm.eu 